\title{
Definition of Laplace Transforms for Distribution of the First Passage of Zero Level of the Semi-Markov Random Process with Positive Tendency and Negative Jump
}

\author{
Tamilla I. Nasirova, Ulviyya Y. Kerimova \\ Baku State University, Baku, Azerbaijan \\ E-mail: shaxbazi_a@yahoo.com, ulviyye_kerimova@yahoo.com \\ Received May 5, 2011; revised May 26, 2011; accepted May 29, 2011
}

\begin{abstract}
One of the important problems of stochastic process theory is to define the Laplace transforms for the distribution of semi-markov random processes. With this purpose, we will investigate the semi-markov random processes with positive tendency and negative jump in this article. The first passage of the zero level of the process will be included as a random variable. The Laplace transforms for the distribution of this random variable is defined. The parameters of the distribution will be calculated on the basis of the final results.
\end{abstract}

Keywords: Laplace Transforms, Semi-Markov Random Process, Random Variable, Process With Positive Tendency And Negative Jumps

\section{Introduction}

There are number of works devoted to definition of the Laplace transforms for the distribution of the first passage of the zero level. (Borovkov 2004) [1] defined the explicit form of the distribution, while (Klimov 1996) [2] and (Lotov V. I.) [3] indicated implicit form of the distribution of the first passage of zero level. The presented work explicitly defines the Laplace transforms for the unconditional and conditional distribution of the semi-markov random processes with positive tendency and negative jump.

\section{Problem}

Let's assume that $\xi_{k}$ and $\zeta_{k}, k \geq 1$ random variables of independent $\left\{\xi_{k}, \zeta_{k}\right\}_{k \geq 1}$ random variable sequence evenly distributed in $\{\Omega, F, P(\cdot)\}$ probability face are given. Using these random variables we will derive the following semi-markov random process:

if

$$
X(t)=z+t-\sum_{i=1}^{k-1} \zeta_{i},
$$

$$
\sum_{i=1}^{k-1} \xi_{i} \leq t<\sum_{i=1}^{k} \xi_{i} k=\overline{1, \infty}
$$

$X(t)$ process is the (asymptotic) semi-markov random processes with positive tendency and negative jump.
Let's include the $\tau_{1}^{0}$ random variable defined as below:

$$
\tau_{1}^{0}=\min \{t: X(t) \leq 0\}
$$

where $\tau_{1}^{0}$, is the time of the first passage of $X(t)$ process.

We need to find Laplace transform for distribution of $\tau_{1}^{0}$ random variable.

\section{Definition of Laplace Transform for the Distribution of $\tau_{1}^{0}$ Random Variable}

Let us set Laplace transform for the distribution of $\tau_{1}^{0}$ random variable as $L(\theta)$ :

$$
\begin{aligned}
& L(\theta)=E e^{-\theta \tau_{1}^{0}}, \theta>0, \\
& L(\theta \mid z)=E\left(e^{-\theta \tau_{1}^{0}} \mid X(0)=z\right), z \geq 0
\end{aligned}
$$

In this case we can express the equation as

$$
\tau_{1}^{0}= \begin{cases}\xi_{1,} & z+\xi_{1}-\zeta_{1}<0, \\ \xi_{1}+T, & z+\xi_{1}-\zeta_{1}>0,\end{cases}
$$

Thus, $T$ and $\tau_{1}^{0}$ are evenly distributed random variables.

Our goal is to find Laplace transform of relative and non-relative distribution of $\tau_{1}^{0}$ random variable.

According to the formula of total probability, we can put it as 


$$
E\left(e^{-\theta \tau_{1}^{0}} \mid X(0)=z\right)=\int_{\Omega} e^{-\tau_{1}^{0}} P(\mathrm{~d} \omega)=\int_{\left\{\omega: z+\xi_{1}-\zeta_{1}<0\right\}} e^{-\theta \xi_{1}} P(\mathrm{~d} \omega)+\int_{\left\{\omega: z+\xi_{1}-\zeta_{1}>0\right\}} e^{-\theta\left(\xi_{1}+T\right)} P(\mathrm{~d} \omega)
$$

If to consider the following substitution

$$
\xi_{1}=s ; s_{1}=y ; T=\beta
$$

we derive

$$
\begin{aligned}
& E\left(e^{-\theta \tau_{1}^{0}} \mid X(0)=z\right)=\int_{s=0}^{\infty} \int_{y=z+s}^{\infty} e^{-\theta s} P\left\{\xi_{1} \in \mathrm{d} s ; \zeta_{1} \in \mathrm{d} y\right\} \\
& +\int_{s=0}^{\infty} \int_{y=0}^{z+s} \int_{\beta=0}^{\infty} e^{-\theta(s+\beta)} P\left\{\xi_{1} \in \mathrm{d} s ; \zeta_{1} \in \mathrm{d} y ; T \in \mathrm{d} \beta\right\} \\
& =\int_{s=0}^{\infty} e^{-\theta s} P\left\{\xi_{1} \in \mathrm{d} s\right\} \int_{y=z+s}^{\infty} P\left\{\zeta_{1} \in \mathrm{d} y\right\} \\
& +\int_{s=0}^{\infty} e^{-\theta s} \int_{y=0}^{z+s} \mathrm{~d} P\left\{\zeta_{1}<y\right\} \mathrm{d} P\left\{\xi_{1}<s\right\} L(\theta \mid z+s-y) \\
& =\int_{s=0}^{\infty} e^{-\theta s} P\left\{\xi_{1} \in \mathrm{d} s\right\} \mathrm{P}\left\{\zeta_{1}>z+s\right\} \\
& +\int_{s=0}^{\infty} e^{-\theta s} \int_{\beta=z+s}^{0} L(\theta \mid \beta) \mathrm{d} P\left\{\zeta_{1}<z+s-\beta\right\} \mathrm{d} P\left\{\xi_{1}<s\right\}
\end{aligned}
$$

or

$$
\begin{aligned}
& L(\theta \mid z)=\int_{s=0}^{\infty} e^{-\theta s} P\left\{\zeta_{1}>z+s\right\} P\left\{\xi_{1} \epsilon \mathrm{d} s\right\} \\
& +\int_{s=0}^{\infty} e^{-\theta s} \int_{y=0}^{z+s} L(\theta \mid z+s-y) \times P\left\{\zeta_{1} \epsilon \mathrm{d} y\right\} P\left\{\xi_{1} \epsilon \mathrm{d} s\right\}
\end{aligned}
$$

Let's assume that $z+s-y=\alpha$. In this case we will receive the following integral equation:

$$
\begin{aligned}
& L(\theta \mid z)=\int_{s=0}^{\infty} e^{-\theta s} P\left\{\zeta_{1}>z+s\right\} P\left\{\xi_{1} \epsilon \mathrm{d} s\right\} \\
& -\int_{s=0}^{\infty} e^{-\theta s} \int_{\propto=0}^{z+s} L(\theta \mid \alpha) \mathrm{d}_{\propto} P\left\{\zeta_{1}<z+s-\propto\right\} \mathrm{d} P\left\{\xi_{1} \epsilon \mathrm{d} s\right\}
\end{aligned}
$$

We will solve this integral equation in special case.

Let's assume that $\xi_{1}$ random variable has the Erlangian distribution of $\mathrm{m}$ construction, while $\zeta_{1}$ random variable has the single construction Erlangian distribution:

$$
\begin{aligned}
& P\left\{\xi_{1}(\omega)<t\right\} \\
& =\left\{1-\left[1+\lambda t+\frac{(\lambda t)^{2}}{2 !}+\cdots+\frac{(\lambda t)^{m-1}}{(m-1) !}\right] e^{-\lambda t}\right\} \varepsilon(t), \quad t>0, \\
& P\left\{\zeta_{1}(\omega)<t\right\}=\left[1-e^{-\mu t}\right] \varepsilon(t), \\
& t>0 \text {. }
\end{aligned}
$$

where

$$
\varepsilon(t)=\left\{\begin{array}{l}
0, t<0 \\
1, t>0
\end{array}\right.
$$

In this case Equation (2) will be as follows:

$$
\begin{aligned}
& L(\theta \mid z)=\frac{\lambda^{m}}{(\lambda+\mu+\theta)^{m}} e^{-\mu z} \\
& -\frac{\lambda^{m} \mu}{(m-1) !} e^{-\mu z} \int_{s=0}^{\infty} e^{-(\lambda+\mu+\theta) s} s^{m-1} \int_{\propto=0}^{z+s} e^{\mu \propto} L(\theta \mid \propto) \mathrm{d} \propto \mathrm{d} s
\end{aligned}
$$

We can derive differential equation from this integral equation. For this purpose, we will multiply both sides of Equation (3) by $e^{\mu z}$ :

$$
\begin{aligned}
& e^{\mu z} L(\theta \mid z)=\frac{\lambda^{m}}{(\lambda+\mu+\theta)^{m}} \\
& -\frac{\lambda^{m} \mu}{(m-1) !} \int_{s=0}^{\infty} e^{-(\lambda+\mu+\theta) s} s^{m-1} \int_{\propto=0}^{z+s} e^{\mu \propto} L(\theta \mid \propto) \mathrm{d} \propto \mathrm{d} s
\end{aligned}
$$

If we increment both sides by $z$, we will get:

$$
\begin{aligned}
& e^{\mu z} L(\theta \mid z)+e^{\mu z} L^{\prime}(\theta \mid z) \\
& =-\frac{\lambda^{m} \mu e^{\mu z}}{(m-1) !} \int_{s=0}^{\infty} e^{-(\lambda+\mu+\theta) s} s^{m-1} e^{\mu s} L(\theta \mid z+s) \mathrm{d} s
\end{aligned}
$$

In this case we will receive the differential equation with $(\mathrm{m}+1)$ construction:

$$
\begin{aligned}
& \sum_{k=0}^{m} C_{m}^{k}\left[\mu L^{(k)}(\theta \mid z)+L^{(k+1)}(\theta \mid z)\right] \\
& \times e^{(\lambda+\theta) z}(-1)^{m-k}(\lambda+\theta)^{m-k} \\
& =(-1)^{m} \lambda^{m} \mu e^{-(\lambda+\theta) z} L(\theta \mid z)
\end{aligned}
$$
be

The general solution of this differential equation will

$$
(\theta \mid z)=C_{1}(\theta) L e^{k_{1}(\theta) z} C_{2}(\theta) e^{k_{2}(\theta) z}+\cdots+C_{m}(\theta) e^{k_{m(\theta) z}}
$$

By finding $C_{1}(\theta), \cdots, C_{m}(\theta)$ from Equation (3) we will get the following systematic equations:

$$
\left.\begin{array}{c}
L(\theta \mid 0)=\frac{\lambda^{m}}{(\lambda+\mu+\theta)^{m}}+\frac{\lambda^{m} \mu}{(m-1) !} \int_{s=0}^{\infty} e^{-(\lambda+\mu+\theta \mid) s} \int_{s=0}^{\infty} \times s^{m-1} \int_{\propto=0}^{s} e^{\mu \propto} L(\theta \mid \propto) \mathrm{d} \propto \mathrm{d} s \\
L^{\prime}(\theta \mid 0)=-\mu L(\theta \mid 0)+\frac{\lambda^{m} \mu}{(m-1) !} \int_{s=0}^{\infty} e^{-(\lambda+\theta) s} \times s^{m-1} L(\theta \mid s) \mathrm{d} s \\
\vdots \\
\sum_{k=0}^{m-1} C_{m}^{k}\left[\mu L^{(k)}(\theta \mid 0)+L^{(k+1)}(\theta \mid 0)\right]=(-1)^{m} \lambda^{m} \mu \int_{x=0}^{\infty} e^{-(\lambda+\theta) x} L(\theta \mid x) \mathrm{d} x
\end{array}\right\}
$$


By exploitation of Equation (5), Equation (6) becomes

$$
\left.\begin{array}{c}
\sum_{k=0}^{m} C_{i}(\theta)=\frac{\lambda^{m}}{(\lambda+\mu+\theta)^{m}}+\frac{\lambda^{m} \mu}{(m-1) !} \int_{s=0}^{\infty} e^{-(\lambda+\mu+\theta) s} \times s^{m-1} \int_{\propto=0}^{s} e^{\mu \propto} \sum_{i=1}^{m} C_{i}(\theta) e^{k_{i}(\theta) \alpha} \\
\sum_{i=1}^{m} C_{i}(\theta) k_{i} \theta+\mu \sum_{i=1}^{m} C_{i}(\theta)=\frac{\lambda^{m} \mu}{(m-1) !} \int_{s=0}^{\infty} e^{-(\lambda+\theta) x} \times x^{m-1} \sum_{i=1}^{m} C_{i}(\theta) e^{k_{i}(\theta) x} \mathrm{~d} x \\
\vdots \\
\sum_{k=0}^{m-1} C_{m}^{k}\left[\mu \sum_{i=1}^{m} k^{m} C_{i}(\theta)+k^{m+1} \sum_{i=0}^{m} C_{i}(\theta)\right]=(-1)^{m} \lambda^{m} \mu \int_{x=0}^{\infty} e^{-(\lambda+\theta) x} \sum_{i=1}^{m} C_{i}(\theta) e^{k_{i}(\theta) x} \mathrm{~d} x
\end{array}\right\}
$$

After simplification of the last system we will get

$$
\left.\begin{array}{c}
\sum_{i=1}^{m} C_{i}(\theta)=\frac{\lambda^{m}}{(\lambda+\mu+\theta)^{m}}+\frac{\lambda^{m} \mu}{(m-1) !} \sum_{i=1}^{m} \frac{C_{i}(\theta)}{\mu+k_{i}(\theta)} \frac{(m-1) !}{\left(\lambda+\theta-k_{i}(\theta)\right)^{m}}-\frac{(m-1) !}{(\lambda+\theta+\mu)^{m}} \\
\sum_{i=1}^{m} C_{i} \theta k_{i}\left(\theta+\mu \sum_{i=1}^{m} C_{i}(\theta)\right)=\frac{\lambda^{m} \mu}{(m-1) !} \sum_{i=1}^{m} \frac{(m-1) !}{\left(\lambda+\theta-k_{i}(\theta)\right)^{m}} C_{i} \theta \\
\vdots \\
\sum_{i=1}^{m} C_{m}^{k}\left[\mu \sum_{i=1}^{m} k^{m} C_{i}(\theta)+\sum_{i=1}^{m} k^{m+1} C_{i}(\theta)\right]=(-1)^{m} \lambda^{m} \mu \sum_{i=1}^{m} \frac{C_{i} \theta}{\left(\lambda+\theta-k_{i}(\theta)\right)^{m}}
\end{array}\right\}
$$

or

$$
\left.\begin{array}{c}
\left.\sum_{i=1}^{m}\left[1-\lambda^{m} \mu \frac{(\lambda+\mu+\theta)^{m}-\left(\lambda+\theta-k_{i}(\theta)\right)^{m}}{\left(\mu+k_{i}(\theta)\right)(\lambda+\mu+\theta)^{m}\left(\lambda+\theta-k_{i}(\theta)\right)^{m}}\right] C_{i}(\theta)=\frac{\lambda^{m}}{(\lambda+\mu+\theta)^{m}}\right] C_{i}(\theta)=0 \\
\sum_{i=1}^{m}\left[\mu+k_{i}(\theta)-\frac{\lambda^{m} \mu}{\left(\lambda+\theta-k_{i}(\theta)\right)^{m}}\right] \\
\sum_{k=0}^{m-1} C_{m}^{k} k^{m}\left[\mu+k_{i}(\theta)-\frac{\lambda^{m} \mu}{\left(\lambda+\theta-k_{i}(\theta)\right)^{m}}\right] C_{i}(\theta)=0
\end{array}\right\}
$$

By exploitation of

$$
\lambda^{m} \mu=\left(\mu+k_{i}(\theta)\left(\lambda+\theta-k_{i}(\theta)\right)^{m}\right.
$$

Equation (9) becomes

$$
\left.\begin{array}{c}
\sum_{i=1}^{m}\left[1-\frac{(\lambda+\mu+\theta)^{m}-\left(\lambda+\theta-k_{i}(\theta)\right)^{m}}{(\lambda+\mu+\theta)^{m}}\right] C_{i}(\theta)=\frac{\lambda^{m}}{(\lambda+\mu+\theta)^{m}} \\
\sum_{i=1}^{m}\left[\mu+k_{i}(\theta)-\left(\mu+k_{i}(\theta)\right)\right] C_{i}(\theta)=0 \\
\sum_{k=0}^{m-1} C_{m}^{k} k^{m}\left[\mu+k_{i}(\theta)-\left(\mu+k_{i}(\theta)\right)\right] C_{i}(\theta)=0
\end{array}\right\}
$$

or 


$$
\left.\begin{array}{c}
\sum_{i=1}^{m}\left[\lambda+\theta-k_{i}(\theta)\right]^{m} C_{i}(\theta)=\lambda^{m} \\
\sum_{i=1}^{m} 0 \times C_{i}(\theta)=0 \\
\vdots \\
\sum_{i=1}^{m} 0 \times C_{i}(\theta)=0
\end{array}\right\}
$$

Thus, (9) is a linear dependence equation system, as

$$
\begin{aligned}
& C_{2}(\theta)=C_{3}(\theta)=\cdots=C_{m}(\theta)=0 \\
& C_{1}(\theta)=\frac{\lambda^{m}}{\left[\lambda+\theta-k_{1}(\theta)\right]^{m}}
\end{aligned}
$$

Then the general solution of integral Equation (3) will be as follows:

$$
L(\theta \mid z)=C_{1}(\theta) e^{k_{1}(\theta) z}=\frac{\lambda^{m}}{\left[\lambda+\theta-k_{1}(\theta)\right]^{m}} e^{k_{1}(\theta) z}
$$

This expression is the Laplace transform for relative distribution of $\tau_{1}^{0}$ random variable. Then, we will need to find $L(\theta)$. In accordance with formula of total probability,

$$
L(\theta)=\int_{z=0}^{\infty} L(\theta \mid z) d P\{X(0)<z\}
$$

and as the distribution of $X(0)$ and $\zeta_{1}(\omega)$ random variables are same,

$$
\begin{aligned}
L(\theta) & =\int_{\mathrm{z}=0}^{\infty} C_{1}(\theta) e^{k_{1}(\theta) z} \mathrm{dP}\{X(0)<z\} \\
& =C_{1}(\theta) \int_{\mathrm{z}=0}^{\infty} e^{k_{1}(\theta) z} \frac{\lambda^{m}}{(m-1) !} z^{m-1} e^{-\lambda z} \mathrm{~d} z \\
& =\frac{\lambda^{m} C_{1}(\theta)}{(m-1) !} \int_{z=0}^{\infty} e^{-\left[\lambda-k_{1}(\theta)\right] z} z^{m-1} \mathrm{~d} z \\
& =\frac{\lambda^{m}}{\left[\lambda-k_{1}(\theta)\right]^{m}} C_{1}(\theta)
\end{aligned}
$$

Therefore,

$$
L(\theta)=\frac{\lambda^{m}}{\left[\lambda-k_{1}(\theta)\right]^{m}} C_{1}(\theta)
$$

This expression is the Laplace transform for non-relative distribution of $\tau_{1}^{0}$ random variable.

Respectively, we will get the following characteristics for $\lambda \mathrm{m}>\mu$

$$
\begin{aligned}
& E \tau_{1}^{0}=-L^{\prime}(0)=\frac{m(\lambda+\mu)}{\lambda(\lambda-m \mu)} \\
& L^{\prime}(0)= \frac{m^{3} \mu^{2}(3-m)}{\lambda^{2}(\lambda-m \mu)^{2}}+2 \frac{m^{3} \mu}{\lambda(\lambda-\mu)(\lambda-m \mu)} \\
&+\frac{m(m+1) \lambda}{\left((\lambda-m \mu)^{3}\right.} \\
& D \tau_{1}^{0}= L^{\prime}(0)-\left(L^{\prime}(0)\right)^{2}=\frac{m^{3} \mu^{2}(3-m)}{\lambda^{2}(\lambda-m \mu)^{2}} \\
&+ 2 \frac{m^{3} \mu}{\lambda(\lambda-\mu)(\lambda-m \mu)} \\
&+\frac{m(m+1) \lambda}{(\lambda-m \mu)^{3}}-\frac{m^{2}(\lambda+m \mu)^{2}}{\lambda^{2}(\lambda-m \mu)^{2}} \\
& E\left(\tau_{1}^{0} \mid z\right)=\frac{m(1+z \mu)}{\lambda-m \mu} \frac{m}{(\lambda-m \mu)^{2}}+\frac{m((m+1))(m+z \mu) \mu}{(\lambda-m \mu)^{3}} \\
& D\left(\tau_{1}^{0} \mid z\right)=\frac{(\lambda)}{(\lambda-m)}
\end{aligned}
$$

\section{Conclusions}

In this article we have defined Laplace transforms for relative and non-relative distribution of the first passage of zero level of semi-markov random process with positive tendency and negative jump.

\section{References}

[1] A. A. Borovkov, "On the Asymptotic Behavior of the Distributions of First-Passage," Mathematics and Statistics, Vol. 75, No. 1, 2004, pp. 24-39. doi:10.1023/B:MATN.0000015019.37128.cb

[2] V. I. Lotov, "On the Asymptotics of the Distributions in Two-Sided Boundary Problems for Random Walks Defined on a Markov Chain," Siberian Advances in Mathematics, Vol. 1, No. 3, 1991, pp. 26-51.

[3] G. P. Klimov, "Stochastic Queuening Systems," Nauka, Moscow, 1966.

[4] T. I. Nasirova and R. I. Sadikova, "Laplace Transformation of the Distribution of the Time of System Sojourns within a Band," Automatic Control and Computer Sciences, Vol. 43, No. 4, pp. 190-194. doi:10.3103/S014641160904004X 